\title{
Ortogonale voorstelling van drywing deur middel van kwaternione
}

\author{
J. H. R. Enslin* \\ Dept. Elektriese Ingenieurswese, Universiteit van Pretoria, Pretoria 0002 \\ D. F. van der Merwe \\ Laboratorium vir Energie, Randse Afrikaanse Universiteit, Posbus 524, Johannesburg 2000
}

Ontvang 23 Januarie 1989; anvaar 17 Alugustus 1989

\section{UITTREKSEL}

Die toename in die gehruik van drywingsheheerstelsels, wat die belading-en opwekfunksies van drywing in 'n stelsel vervorm, ten opsigte van 'n eenvoudige sinusodaal, het die aunvaurbaarheid van huidige drywingsdefinisies bevraagteken. Die wiskundige voorstelling van dry'wing deur middel van die kwaternioonteorie w'ord ondersoek om 'n algemene definisie van drywing daar te stel, vir stelsels waar die opwek- en beladingsfinksies nie eenvoudige sinusvormige verlope besit nie. Die algemene beskrywing van drwing word aan die hand van tipiese elektriese stelsels geillustreer, maar word voorgestel in die algemene analise van alle ander drywingstelsels, hetsy meganies, termies of chemies. Drywing word verdeel in komponente wat die netwerk heskrvf aan die hand van energie-oordrag en is onderling ortogonaal. Dic kw'aternioonteorie hesit ortogonale eienskappe wat, uitgande vanaf die Cauchy-Schwarz-ongelykheid, die beladingsfunksie op die opwekfunksie heskryf om sodoende drywing in drie dimensies voor te stel.

\section{ABSTRACT \\ Orthogonal representation of power with the aid of quaternions}

The increased use of power control equipment resulted in the distortion of the excitation and response functions in power sy'stems from simple sinusoids. These systems resulted in discrepancies in the general definition of power. This distortion has a negative effect on the accurate definition and representation of power in a contaminated power system. The mathematical representation of power with the aid of the theory of quaternions in vector calculus is investigated to obtain a generalized definition of power in all power systems, especially in power systems where the excitation and response functions show non-simusoidal characteristics. The general description of power is illustrated with the aid of electrical power systems, but is however proposed to be heneficial in all power systems, being mechanical, thermal or chemical. Power is divided into different orthogonal components which describes the energy transfer through a system. The quaternion theory has orthogonal properties which can be used together with the Cauchy-Schwarz inequality to describe the pow'er components using the excitation and respective response functions.

\footnotetext{
*Outeur aan wie korrespondensie gerig kan word.
} 


\section{INLEIDING}

Oneffektiewe benutting van die infrastruktuur om drywing van een punt in 'n stelsel na 'n ander oor te dra, is lank reeds bekend. ${ }^{2.6}$ Dit is egter tans steeds 'n probleem om die drywingsvloei te analiseer en verder vir die oneffektiewe benutting te kompenseer, wanneer die verlope van die opwek- en responsiefunksies nie deur eenvoudige periodiese en sinusvormige funksies beskryf kan word nie. 3.4 .5 In drywingstelsels, veral in elektriese drywingstelsels, word vanaf die begin van die eeu fasors van die opwek- en responsiefunksies gebruik om drywingsvloei te beskryf. Die voordele van die fasorteorie is legio. Die teorie is oor die algemeen eenvoudig en is maklik bruikbaar om drywingsvloei te bereken, aangesien die tydfunksies van die opwek- en responsiefunksies beskryf word in terme van slegs 'n grootte en 'n fasehoek. Die voorwaarde geld natuurlik dat sowel die opwek- as responsiefunksies sinusvormige tydsverlope moet besit, wat fundamentele beperking in die gebruik van dié teorie in algemene drywingstelsels impliseer.

Deur gebruik te maak van die fasorteorie, ontwikkel deur Steinmetz, word alle sinusvormige seine deur middel van Euler se reël getransformeer na die eksponensiële voorstelling in ' $n$ reële kompenent en 'n imaginêre komponent:

$\mathbf{R}=\mathrm{R}^{\mathrm{J} \varphi}=\mathrm{R} \cdot \cos \varphi+\mathrm{j} \mathrm{R} \cdot \sin \varphi=\mathrm{R} / \underline{\varphi^{\circ}}$

Dit is duidelik dat bogenoemde fasorbeskrywing interessant genoeg die som van 'n reële getal en 'n imaginêre getal is, of wisk undig die som van 'n skalaar $R \cdot \cos \varphi$ en ' $n$ vektor $\mathbf{j} R \cdot \sin \varphi$ met $\mathbf{j}$ 'n translasie deur $90^{\circ}$ $(\mathbf{j} \equiv \underline{\underline{90}}$ ).

\section{WISKUNDIGE VOORSTELLING VAN SINUS- VORMIGE SPANNINGS EN STROME}

In die voorstelling van drywingsvloei deur ' $n$ elektriese netwerk word gewoonlik van die fasorvoorstelling van die spanning en stroom gebruik gemaak. 'n Effek tiewe waarde en fasehoek word gebruik om die golfvorms tydinvariant te beskryf. Soos hierbo aangetoon, lewer die fasoranalise egter probleme in nie-lineêre en tydsafhanklike sisteme waar aperiodiese en nie-sinusvormige golfvorms vir stroom en spanning voorkom.

Met spanning as die verwysing, is die fasorvoorstelling van spanning en stroom

$$
\begin{array}{rlrl}
\mathbf{U} & =\mathrm{U}_{\mathrm{en}} \mathrm{e}^{\mathrm{j} \omega \omega t} & \mathbf{I} & =\mathrm{I}_{\mathrm{en}} \mathrm{e}^{(j \omega t-j \theta)} \\
& =\mathrm{U}_{\mathrm{eff}} \underline{0}^{\circ} & & =\mathrm{I}_{\mathrm{err}} / \underline{\Theta}^{\circ}
\end{array}
$$

waar $\mathbf{U}_{\text {eff }}$ en $I_{\text {eff }}$ die effek tiewe waardes van die fasors $\mathbf{U}$ en $\mathbf{I}$ is, en $\Theta$ die fasehoek tussen $\mathbf{U}$ en $\mathbf{I}$ verteenwoordig. Die komplekse drywing $\mathbf{S}$ word dan gegee deur

$\mathbf{S}=\mathbf{U}^{*}=\mathbf{P}+\mathbf{j} Q(\mathbf{j}=v-1)$

$P=U_{\text {eff }} I_{\text {eff }} \cos \Theta$ en $Q=U_{\text {eff }} I_{\text {elf }} \sin \Theta$

P staan bekend as die aktiewe drywing, terwyl $Q$ as die reaktiewe of fiktiewe drywing bekend staan. Om die verskillende drywingskomponente, asook die ortogonaliteit tussen die komponente te toon, kan 'n mens van kwaternione' gebruik maak.

\section{KENMERKE VAN KWATERNIONE}

'n Kwaternioon' $\mathbf{q}=(\mathrm{a}, \mathrm{b}, \mathrm{c}, \mathrm{d})$, word gedefinieer as die som van 'n skalaar en 'n vektor met

$\mathbf{q}=a \mathbf{i}+b \mathbf{j}+\mathbf{c k}+\mathbf{d}$

waar a, b, c, d reële getalle is in ' $n$ bepaalde volgorde, en $\mathbf{l}, \mathbf{i}, \mathbf{j}, \mathbf{k}$ kwaterniooneenhede ${ }^{1}$ is.

$\mathrm{I}=(1,0,0,0) ; \quad \mathbf{i}=(0,1,0,0) ; \quad \mathbf{j}=(0,0,1,0)$;

$\mathbf{k}=(0,0,0,1)$

Vermenigvuldiging gehoorsaam die volgende reëls:

$\mathbf{i}^{2}=\mathbf{j}^{2}=\mathbf{k}^{2}=-\mathbf{l} ; \quad \mathbf{i j}=\mathbf{k}=-\mathbf{j i} ; \quad \mathbf{j k}=\mathbf{i}=-\mathbf{k j} ;$

$\mathbf{k i}=\mathbf{j}=-\mathbf{i k}$

Verder word 'n vektordeel V(q), 'n skalaardeel $\mathbf{S}(\mathbf{q})$, 'n norm $\mathrm{N}(\mathbf{q})$, resiproke $\mathbf{q}^{\prime}$ en gekonjugeerde $\mathbf{q}^{*}$ van kwaternioon gedefinieer:

$\mathrm{V}(\mathbf{q})=\mathrm{Vq}=\mathrm{a} \mathbf{i}+\mathrm{b} \mathbf{j}+\mathbf{c k} ; \mathrm{S}(\mathbf{q})=\mathrm{Sq}=\mathrm{d}$

sodat $\mathbf{q}=\mathbf{V q}+\mathrm{Sq}$ en $\mathbf{q}^{*}=-\mathbf{V q}+\mathrm{Sq}$

$\mathbf{N}(\mathbf{q})^{2}=\mathbf{q q}^{*}=\mathrm{a}^{2}+\mathrm{b}^{2}+\mathrm{c}^{2}+\mathrm{d}^{2}$ en $\mathbf{q}^{\prime}=\mathbf{q}^{*} / \mathbf{N}(\mathbf{q})$

Die produk $\mathbf{q . q ^ { 1 }}$ van twee kwaternione kan geskryf word as

$$
\begin{aligned}
& \mathbf{q} \cdot \mathbf{q}^{1}=d^{1}-a a^{1}-b b^{1}-c c^{1}+d\left(a^{1} \mathbf{i}+b^{1} \mathbf{j}+c^{1} \mathbf{k}\right) \\
& +d^{1}(a \mathbf{i}+b \mathbf{j}+c \mathbf{k}) \\
& +\left|\begin{array}{lll}
\mathbf{i} & \mathbf{j} & \mathbf{k} \\
\mathrm{a} & \mathrm{b} & \mathrm{c} \\
\mathrm{a} & \mathrm{b}^{1} & \mathrm{c}^{\mathrm{i}}
\end{array}\right| \\
& =S \mathbf{q} \mathbf{q}^{1}+S \mathbf{q} V \mathbf{q}^{1}+S \mathbf{q}^{1} V \mathbf{q}+V \mathbf{q} V \mathbf{q}^{1}
\end{aligned}
$$

\section{VOORSTELLING VAN DRYWING D.M.V. KWATERNIONE}

Nominaal kan 'n spanning en 'n stroom as kwaternione geskryf word

$$
\begin{aligned}
& \mathbf{U q}=U_{1}+U_{1} \mathbf{i}+U_{j} \mathbf{j}+U_{k} \mathbf{k} \\
& I^{q}=I_{1}+I_{1} \mathbf{i}+I_{1} \mathbf{j}+I_{k} \mathbf{k}
\end{aligned}
$$

Oor die algemeen sal 'n sinusvormige spanning en stroom oor 'n periode twee komponente (en nie vier nie) besit, gegewe dat die gemiddelde waarde nul is. Die gemiddelde waardes word as $U_{1}$ en $I_{1}$ assosieer, terwyl die spanning- en stroomvektor uit slegs twee komponente bestaan, $U_{i} ; I_{i}$ en $U_{j} ; I_{j}$. So kan dan analoog aan die fasorvoorstelling, die kwaternioondrywing definieer word as

$\mathbf{S q}=\mathrm{UqIq}^{*}$

$\mathbf{S q}=\left(\mathbf{U}_{\mathrm{i}} \mathbf{i}+\mathbf{U}_{\mathrm{j}} \mathbf{j}\right)\left(-\mathrm{l}_{1} \mathbf{i}-\mathrm{I}_{\mathrm{j}} \mathbf{j}\right)$ waar die ander komponente nul is.

Met die fasorvoorstelling neem ons die gemiddelde drywing oor een periode. om 'n ekwivalente vergelyk te tref tussen die twee voorstellings (fasor en kwaternioon). 


$$
\begin{aligned}
\mathbf{S}= & \left(U_{i} I_{1}+U_{j} I_{i}\right)+\left(U_{j} I_{i}-U_{i} I_{j}\right) \bar{k} \\
& \text { of meer algemeen: } \\
\mathbf{S}= & P+D_{i} \mathbf{i}+D_{j} \mathbf{j}+D_{k} \mathbf{k}
\end{aligned}
$$

Die aktiewe drywing is $U_{1} I_{1}+U_{1} I_{1}$, terwyl slegs een ander komponent $D_{k}$ die reaktiewe komponen voorstel.

$$
\begin{aligned}
\mathbf{S} & =\mathrm{P}+\mathrm{Q} \mathbf{k}=\mathbf{U} \cdot \mathbf{I}+\mathbf{U} \times \mathbf{I} \\
& =\mathrm{U}_{\mathrm{efr}} \mathrm{I}_{\mathrm{efr}} \cos \Theta+\mathbf{k} \mathrm{U}_{\mathrm{efr}} \mathrm{I}_{\mathrm{eff}} \sin \Theta
\end{aligned}
$$

waar $\mathbf{U}$ en I nou die spanning en stroom as vektore voorstel en $\Theta$ die fasehoek tussen $\mathbf{U}$ en $\mathbf{I}$ is.

In meer komplekse golfvorms waar die stroom en spanning nie eenvoudige sinusverlope besit nie, sou die kwaternioondrywing uit meer komponente bestaan het. Die verdere doel is om te toon dat die komponente ortogonaal is. Uit die definisie van 'n kwaternioon volg dat:

$$
\mathrm{N}(\mathrm{Sq})^{2}=\mathrm{P}^{2}+\mathrm{D}_{1}{ }^{2}+\mathrm{D}_{\mathrm{j}}{ }^{2}+\mathrm{D}_{\mathrm{k}}{ }^{2}
$$

wat impliseer dat die komponente wel ortogonale eienskappe besit.

Beskou die volgende voorstelling van die kwaternioonkomponente van stroom en spanning. Neem die effektiewe waardes as $U$ en I van die vektorgedeeltes $\operatorname{van} \mathbf{U q}$ en Iq.

$\mathbf{U q}=\mathrm{U}_{1}+\mathrm{U} \cdot \cos \alpha_{\mathrm{u}} \cdot \cos \beta_{\mathrm{u}} \mathbf{i}+\mathrm{U} \cdot \sin \alpha_{\mathrm{u}} \cdot \cos \beta_{\mathrm{u}} \mathbf{j}$

+ U.sin $\beta_{u} k$

$\mathbf{I q}=I_{1}+I \cdot \cos \alpha_{1} \cdot \cos \beta_{1} \mathbf{i}+1 \cdot \sin \alpha_{1} \cdot \cos \beta \mathbf{j}+I \cdot \sin \beta_{1} \mathbf{k}(16)$

Figuur I toon die vektorkomponente van bostaande kwaternioonvoorstellings. Die spanning en -stroom word aangedui as drie dimensionele vektore. Die reële gedeelte (skalaar) word nie getoon nie, aangesien dit slegs die GS-waarde verteenwoordig en nie 'n aanduiding van rigting gee nie. Die onderlinge hoeke word aangetoon in die figuur.

As die kwaternioondrywing $\mathbf{S q}=\mathbf{U q}_{\mathbf{l q}} *$ in komponentvorm geskryf word, word die volgende uitdrukking verkry soos getoon in bogenoemde figuur.

$\mathbf{S q}=\mathbf{U q} \cdot \mathbf{l q}{ }^{*}$

$$
\begin{aligned}
& S q=U_{1} 1_{1}+U I \cos \alpha_{u} \cdot \cos \beta_{u} \cdot \cos \alpha_{1} \cdot \cos \beta_{i}+U I \cos \beta_{u} \cdot \sin \alpha_{u} \cdot \cos \beta_{1} \cdot \sin \alpha_{1} \\
& +U I \sin \beta_{u} \cdot \sin \beta_{1}+\left(U_{1} I \cos \alpha_{1} \cdot \cos \beta_{1}+I_{1} U \cos \alpha_{u} \cdot \cos \beta_{u}\right. \\
& \left.\quad U I \cos \beta_{u} \cdot \sin u_{u} \cdot \sin \beta_{1}+U 1 \sin \beta_{u} \cdot \cos \beta_{1} \cdot \sin \alpha_{1}\right) \dot{I}+1 U_{1} I \sin \alpha_{1} \cdot \cos \beta_{1} \\
& \left.+I_{1} U \sin \alpha_{u} \cdot \cos \beta_{u}+U 1 \sin \beta_{1} \cdot \cos \beta_{11} \cdot \cos \alpha_{u} \quad U I \sin \beta_{u} \cdot \cos \beta_{1} \cdot \cos \alpha_{1}\right) \mathbf{j} \\
& +\left(-U_{1} I \sin \beta_{1}+I_{1} U \sin \beta_{u} \quad U I \cos \beta_{1} \cos \beta_{u} \cdot \cos \alpha_{u} \cdot \sin \alpha_{i}\right. \\
& \left.+U I \cos \beta_{u} \cdot \cos \beta_{1} \cdot \cos \alpha_{1} \cdot \sin \alpha_{u}\right) k
\end{aligned}
$$

Die kwaternioon $\mathbf{q}=\mathbf{a}+\mathbf{i b}+\mathbf{j c}+\mathrm{d} \mathbf{k}$ kan eentot-een afgebeeld word op:

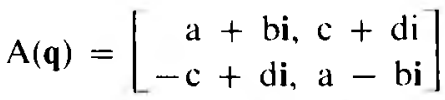

(Let op dat $\mathbf{i}=\sqrt{ }-1$ )

Hierdie voorstelling mag die gebruik van 'n kwaternioon as 'n assosiasie met twee komplekse getalle aanvaarbaarder maak. Nou geld

$\mathrm{A}\left(\mathbf{q}_{1} \mathbf{q}_{2}\right)=\mathrm{A}\left(\mathbf{q}_{1}\right) \mathrm{A}\left(\mathbf{q}_{2}\right)$, ens.

\section{VOORBEELDE MET VERSKILLENDE STROME EN SPANNINGS}

Nou word bogenoemde kwaterioonteorie ondersoek vir voorbeelde waar die stroom en spanning beskryf kan word as periodiese funksies met Fourieruitbreidings.

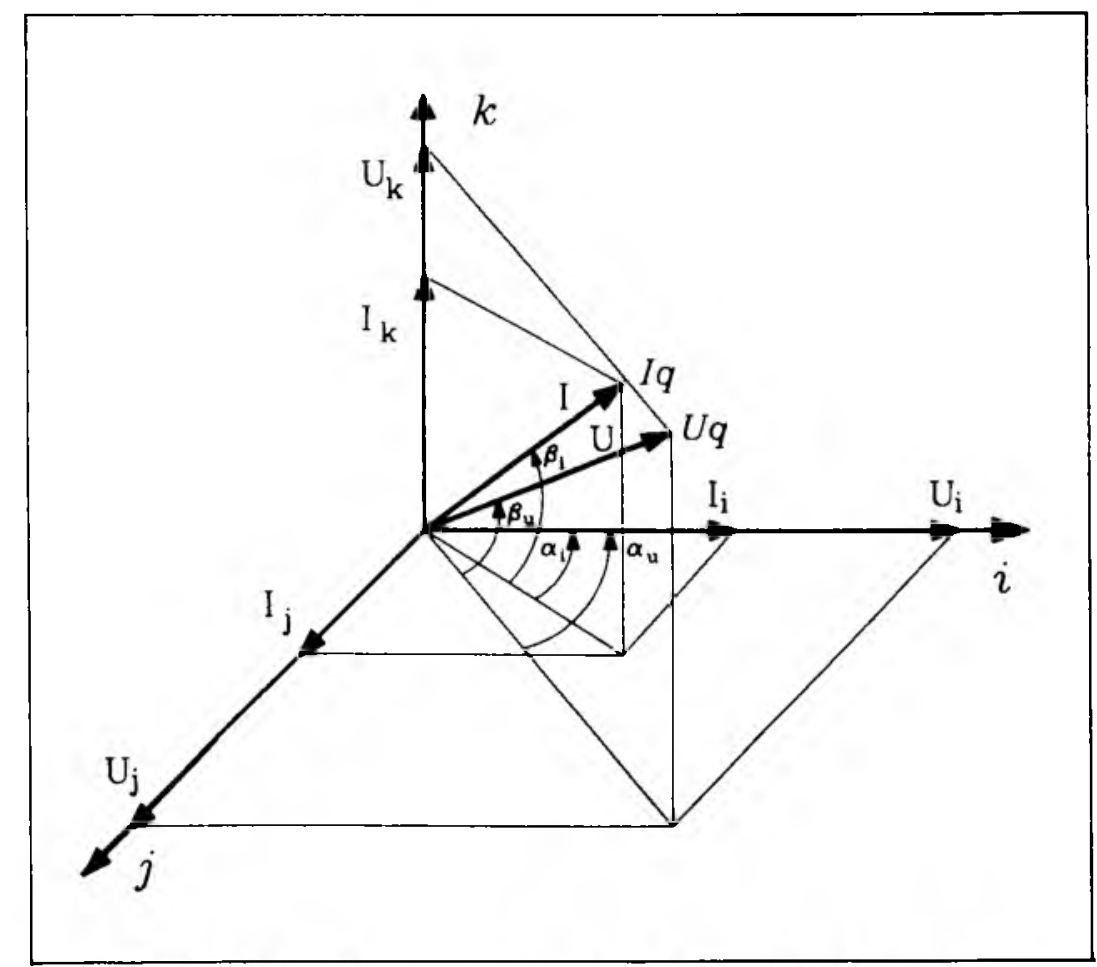

FIGUUR 1: Vektorvoorstelling van kwaternione $\mathbf{U} q$ en $\mathbf{I} q$. 


\section{Voorbeeld I}

'n Spanning en stroom met sinusvormige verlope kan soos volg geskryf word:

$\mathbf{U q}=\mathrm{U} \cos \alpha_{\mathrm{u}} \mathbf{i}+\mathrm{U} \sin \alpha_{\mathrm{u}} \mathbf{j}$

$\mathbf{I q}=\mathrm{I} \cos \alpha_{\mathrm{i}} \mathbf{i}+\mathrm{I} \sin \alpha_{\mathrm{i}} \mathbf{j}$

As $\mathbf{S q}=\mathrm{UqIq}^{*}$ evalueer word, word die volgende verkry:

met $\alpha_{u}=0$.

$\mathbf{S q}=\mathrm{UI} \cos \alpha_{\mathrm{i}}-\mathrm{UI} \sin \alpha_{\mathrm{i}} \mathbf{k}=\mathrm{P}+\mathrm{Q} \mathbf{k}$

wat klop met die bekende komplekse voorstelling van drywing.

\section{Voorbeeld 2}

Neem die spanning as sinusvormig en die stroom as 'n sinus van dieselfde frekwensie as die spanning met 'n derde bofrekwensie.

$\mathrm{u}(\mathrm{t})=\sqrt{ } 2 . \mathrm{U} \cos \omega \mathrm{t}$

$i(t)=\sqrt{ } 2 . I_{1} \cos \left(\omega t-\alpha_{1}\right)+\sqrt{ } 2 . I_{3} \cos \left(3 \omega t-\alpha_{3}\right)(22)$

$I=\sqrt{ }\left(I_{1}^{2}+I_{3}^{2}\right)$

$\mathrm{Uq}=\mathrm{U} \mathbf{i}$

$\mathbf{I q}=\left(\mathrm{I} \cos \beta_{\mathrm{i}} \cdot \cos \alpha_{\mathrm{i}}\right) \mathbf{i}+$

$\left(I \cos \beta_{i} \cdot \sin \alpha_{i}\right) \mathbf{j}+\left(1 \sin \beta_{i}\right) \mathbf{k}$

$\mathrm{Sq}=\mathrm{UI} \cos \beta_{\mathrm{i}} \cos \alpha_{\mathrm{i}}+\mathrm{UI} \sin \beta_{\mathrm{i}} \mathrm{j}$

$-U I \cos \beta_{i} \cdot \sin \alpha_{i} k$

met $\cos \beta_{i}=I_{1} / I ; \sin \beta_{i}=I_{3} / I$ en $\alpha_{1}=\alpha_{1}$ is

$\mathrm{P}=\mathrm{UI}_{1} \cos \alpha_{1}[\mathrm{~W}] ; \mathrm{Q}=\mathrm{UI}_{1} \sin \alpha_{1}[\mathrm{VA}]$;

$\mathrm{D}=\mathrm{UI}_{3}[\mathrm{VA}]$ met $\cos ^{2} \beta_{1}+\sin ^{2} \beta_{1}=1$

Bogenoemde voorbeeld toon die onderlinge verband tussen die drywingskomponente aan. Die komponent D kom slegs voor indien die golfvorm van die spanning en die stroom verskillend is. Figuur 2 toon 'n skematiese voorstelling van die verskillende drywingskomponente van die kwaternioondrywing.

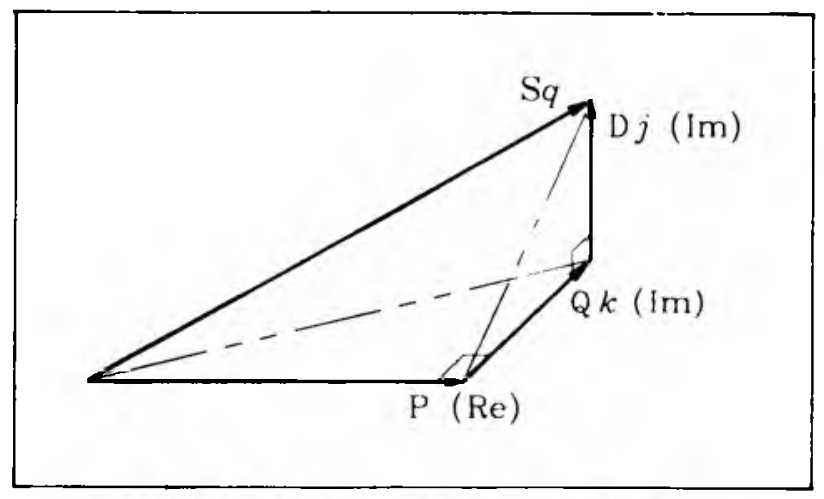

FIGUUR 2: Skematiese voorstelling van kwaternioondrywing $\mathbf{S} q$.

\section{Voorbeeld 3}

Deur gebruik te maak van 'n sinusvormige spanning en 'n blokgolf vir die stroom word die kwaternioondrywing bereken.

$\mathrm{u}(\mathrm{t})=\sqrt{ } 2 . \mathrm{U} \cos \mathrm{wt}$

$\mathrm{i}(\mathrm{t})=+\hat{I}$ vir $\mathrm{O}<\mathrm{t} \leq \mathrm{T} / 2$ \{met die fundamentele faseverskuiwing

$$
=-\hat{\mathrm{I}} \operatorname{vir} \mathrm{T} / 2<\mathrm{t} \leq \mathrm{T}
$$

Die berekening van die kwaternioondrywing uit vergelyking (17) lewer die volgende:

$$
\begin{aligned}
& \mathbf{U q}=\left(\mathrm{U} \cos \alpha_{\mathrm{u}}\right) \mathbf{i}+\left(\mathrm{U} \sin \alpha_{\mathrm{u}}\right) \mathbf{j} \\
& \text { Iq }=\left(I \cos \beta_{1} \cdot \cos \alpha_{1}\right) \mathbf{i}+\left(I \cos \beta_{i} \cdot \sin \alpha_{1}\right) \mathbf{j}+ \\
& \left(I \sin \beta_{1}\right) k \\
& \mathrm{Sq}=\mathrm{UI} \cos \beta_{\mathrm{i}} \cdot \cos \alpha_{\mathrm{i}}+\mathrm{UI} \sin \beta_{\mathrm{i}} \mathrm{j} \\
& \text { - UI } \cos \beta_{1} \cdot \sin \alpha_{i} \mathbf{k} \\
& \text { met } \cos \beta_{\mathrm{i}}=\mathrm{I}_{1} / \mathrm{I}=\sqrt{ } 8 / \pi \text {; } \\
& \sin \beta_{1}=\sqrt{ }\left\{\Sigma\left(I_{n}^{2}\right)\right\} / I=\sqrt{ }\left[1-8 / \pi^{2}\right] \text {; } \\
& \{\mathrm{n}=3,5,7 \ldots \infty\} \text { is } \\
& \mathrm{p}=\sqrt{ } 8 / \pi \text {.UI } \cos \alpha_{1} ; \mathrm{Q}=\sqrt{ } 8 / \pi \text {.UI } \sin \alpha_{1} \text { en } \\
& \mathrm{D}=v^{\prime}\left[1-8 / \pi^{2}\right] . \mathrm{UI} \text {; }
\end{aligned}
$$

Die tekens van die kwaternioondrywing lewer inligting oor die rigting van drywingsvloei. Aktiewe drywing word gedissipeer in die lasweerstand, daarom 'n positiewe teken. Die drywingskomponent $\mathrm{D}$ word die deaktiewe drywing genoem en word verder bespreek in die verwysings $(7,8,9,10)$.

Bogenoemde voorbeelde kan ook op ander maniere bereken word om die akkuraatheid van die kwaternioonvoorstelling te bekragtig.

\section{OPSOMMEND}

Die kwaternioonvoorstelling van drywing is ondersoek nadat die onsekerhede in verband met drywingskomponente met nie-sinusvormige en aperiodiese spannings en strome in nie-lineêre netwerke in die literatuur beklemtoon is. ${ }^{6.8 .9}$ Hierdie voorstelling lei op 'n direkte manier na uitdrukkings vir die drywingskomponente wat verder die regte ortogonaliteitseienskappe besit. Die uitbreding na nie-sinusvormige golfvorms deur middel van Fourieranalise is direk. Sekere voorbeelde van stroom en spanning is ondersoek en blyk akkuraat te wees, indien dit vergelyk word met berekenings d.m.v. ander tegnieke. Dit is interessant dat fiktiewe drywing ook in meganiese sisteme voorkom hoewel verwysings daarna baie beperk is. ${ }^{2}$

\section{VERWYSINGS}

1. Hardy, A.S. (1881). Elements of Quaternions (Ginn \& Co., Boston MA.).

2. Bonnefille, R. (1976). Les realisations d'Electricite de France concernant lénergie marémotrice. La Honille Blanche, 2, 87-149.

3. Depenbrock, M. (1979). Wirk- und Blindleistungen periodisher Ströme in Ein- und Mehrphasensystemen mit periodischen Spannungen beleibiger Kurvenform. VDE-Verlag. GmbHBerlin, ETG-Fachberichte, nr. 6. Vorträge der ETG-Fachtagung. Blindleistung. Aachen. 17-62, 1-2 Oktober.

4. Kusters, N.L. \& Moore, W.J.M. (1980). On the definition of reactive power under nonsinusoidal conditions, IEEE Trans. Power Appl. Sist., vol.PAS-99. no. 5, 1845-1854.

5. Nowomiejski, Z. (1981). Generalized theory of electric power, Archiv fiir Electrolechnik, vol. 63, pp. 177-182.

6. Arrillaga. J., Bradley, D.A.\& Bodger. P.S. (1985). Power sistem harmonics (John Wiley \& Sons).

7. Enslin. J.H.R. \& Van Wyk, J.D. (1988). A new control philosophy for power electronic converters as tictitious power compensators, Proceedings of 19 /h IEEE Poncer Electronics Specialists Conference (PESC-88), Kyoto, Japan, $1188-1196$.

8. Enslin, J.H.R. (1988). Determination and dynamic compensation of fictitious power in electric power systems, D. Ing-Proefskrif, Laboratorium vir Energic, Fakulteit Ingenieurswese. RAU.

9. Enslin. J.H.R. \& Van Wyk, J.D. (1988). Measurement and compensation of fictitious power under nonsinusoidal voltage and current conditions, IEEE Trans. Instrum. Meas., vol. 37, no. 3, 403-408

10 Enslin. J.HI.R. \& Van Wyk, J.D. (1988). 'n Nuwe benadering tot die definisic van elektriese drywing. onder toestande van vervormde spannings en strome, S.A. Tydskrif vir Natumrwetenskap en Tegnologie, vol. 7, no. 2, 76-82. 